\title{
无线局域网EDCA机制MAC接入延时分析
}

\author{
周新运 $1,2+$, 皇甫伟 1,2 , 孙利民 1,2 , 王显雷 2 , 李凯慧 ${ }^{2}$ \\ ${ }^{1}$ (中国科学院 软件研究所 信息安全国家重点实验室,北京 100190) \\ ${ }^{2}$ (中国科学院 研究生院, 北京 100049)
}

\section{MAC Access Delay Analysis of EDCA Mechanism of Wireless LANs}

\author{
ZHOU Xin-Yun ${ }^{1,2+}, \quad$ HUANGFU Wei ${ }^{1,2}, \quad$ SUN Li-Min ${ }^{1,2}, \quad$ WANG Xian-Lei ${ }^{2}, \quad$ LI Kai-Hui ${ }^{2}$ \\ ${ }^{1}$ (State Key Laboratory of Information Security, Institute of Software, The Chinese Academy of Sciences, Beijing 100190, China) \\ ${ }^{2}$ (Graduate University, The Chinese Academy of Sciences, Beijing 100049, China) \\ + Corresponding author: E-mail: xinyun01@ios.cn, http://www.ios.ac.cn
}

Zhou XY, Huangfu W, Sun LM, Wang XL, Li KH. MAC access delay analysis of EDCA mechanism of wireless LANs. Journal of Software, 2008,19(8):2127-2139. http://www.jos.org.cn/1000-9825/19/2127.htm

\begin{abstract}
A model is proposed to analyze the MAC (medium access control) access delay of EDCA (enhanced distribution channel access) mechanism of wireless LANs (local area networks), which is based on the moment generating function and signal flow graph. The model gives the probability distribution function (PDF) of MAC access delay and several relative characteristic numbers, including the mean value, variance, standard deviation and deviation coefficient of MAC access delay. The results of the analytical model coincide with simulations, which validates the model proposed. A lot of analyses are presented. Firstly, the model presents the relationship among number of nodes and sending probability, collision probability, freezing probability and mean delay of all ACs (access categories). Secondly, the model discloses the composition of MAC access delay. Thirdly, the model presents the effect of different values of AIFS (arbitration inter frame spacing) on MAC access delay. Lastly, the model presents that the deviation coefficient of MAC access delay is larger than 1, so when the exponential distribution is adopted as the distribution of MAC access delay, the capacity of the queue system will be evaluated wrongly.
\end{abstract}

Key words: IEEE 802.11e; EDCA (enhanced distribution channel access); MAC (medium access control) access delay; performance analysis

摘 要: 提出一种分析模型, 基于信号流图和母函数方法分析了无线局域网中 EDCA(enhanced distribution channel access)机制的 MAC(medium access control)接入延时. 得到了 MAC 接入延时的概率分布和相关的数字特征, 包括接入延时的均值、方差、标准差和变异系数.仿真结果与模型的分析结果吻合良好,验证了模型和计算方法的

* Supported by the National Natural Science Foundation of China under Grant Nos.60472076, 60673178, 60434030 (国家自然科学基 金); the National Basic Research Program of China under Grant No.2006CB303007 (国家重点基础研究发展计划(973)); the National High-Tech Research and Development Plan of China under Grant Nos.2006AA01Z218, 2006AA01Z215 (国家高技术研究发展计划 (863))

Received 2006-09-12; Accepted 2007-03-19 
正确性.进一步分析了各类 AC(access category)的发送概率、碰撞概率、挂起概率和延时均值随终端数目变化的规 律; 分析了 MAC 接入延时的成因; 指出了 AIFS(arbitration inter frame spacing)值对 MAC 接入延时的影响; 指出接入 延时的变异系数大于 1 , 因此,采用负指数分布作为 MAC 接入延时的近似分布,会错误地估计系统的服务能力.

关键词: IEEE 802.11e;增强的分布式信道访问(enhanced distribution channel access, 简称 EDCA);MAC(medium access control)接入延时;性能分析

中图法分类号: TP393 文献标识码: A

无线局域网中承载着不同的业务,每种业务有不同的 QoS 需求.对于传统的数据业务,需要保证吞吐量和可 靠传输; 而对于实时业务, 需要保证延时和延时抖动在一定范围之内.IEEE 802.11 协议采用 DCF(distributed coordination function)机制, 所有的终端、所有的业务在竞争信道时都处于相等的地位, 无法提供优先级服务. 为 此, 在 IEEE 802.11 $\mathrm{e}^{[1]}$ 协议中提出了两种新的 MAC(medium access control)层访问机制,增强的分布式信道访问 机制 EDCA(enhanced distribution channel access)与混合协调功能(hybrid coordination function,简称 HCF)控制的 信道访问机制 HCCA(HCF controlled channel access).EDCA 为各种业务提供了优先级服务, 使得实时业务等高 优先级应用可以优先访问信道, 满足应用的 QoS 需求. HCCA 是一种轮询协议. 位于接入点中的混合协调器轮流 查询每个终端,根据为其配置的服务质量, 设置批准其访问媒体的权限.

在无线局域网的性能分析方面已经进行了许多研究.Bianchi ${ }^{[2]}$ 提出了一种使用 Markov 链进行 DCF 机制性 能分析的经典模型,得到了 DCF 在饱和情形下的吞吐量. 随后的研究者对 Bianchi 的工作进行了补充和改 进. $\mathrm{Wu}^{[3]}$ 对文献[2]中的模型作了修正, 在重传次数达到限制之后丢弃数据帧;而在文献[2]中, 产生冲突的数据帧 始终重传,没有次数限制.Xiao ${ }^{[4]}$ 扩展了该 Markov 链来分析具有不同 IFS(inter frame spacing)、竞争窗口初始值 和窗口回退次数的多优先级无线局域网性能.Sheng 在文献[5]中对多类别终端共存的无线局域网实时业务性 能作了详细的分析, 文中对每一个虚拟时隙都采用空闲概率、发送概率和碰撞概率计算均值, 汇总所有时隙的 均值得到总的接入延时均值.

在以往的研究工作中, 对吞吐量的研究比较完善, 但是对 MAC 接入延时的分析都只能获取它的均值, 对 MAC 接入延时的分布特征研究不足,而这些分布特征对于利用排队理论分析系统性能是必需的.完整的描述接 入延时的特性需要获取它的概率分布函数.Zhai 在文献[6]中首次提出使用概率分布函数进行性能分析,但是该 文只针对 DCF 机制进行分析, 在 EDCA 机制下, 不同 AC(access category)共存, $C W_{\min }, C W_{\text {max }}$, 最大回退次数和 AIFS(arbitration inter frame spacing)等参数以及虚拟调度器对系统性能的影响, 还需要进一步的研究. 本文使用 信号流图对 EDCA 的接入过程进行分析, 通过计算得到了 MAC 接入延时的概率分布, 依据该分布, 可以得到诸 如均值和方差等性能指标,对 MAC 接入延时有更深的理解.

本文第 1 节对 EDCA 机制、信号流图和梅森公式作简要介绍. 第 2 节对 EDCA 机制下 MAC 接入延时进行 详细分析, 利用信号流图和梅森公式获得 MAC 接入延时的概率分布函数. 第 3 节通过仿真实验, 统计汇总所得 到的数据,验证模型的正确性. 第 4 节对模型进行展开分析. 第 5 节给出文章的总结.

\section{1 背景知识}

\subsection{EDCA机制}

IEEE 802.11e 协议中提出了 EDCA 机制, 为不同业务类型提供不同的访问优先级, 共定义 8 个用户优先级 UP (user priority), 在 MAC 层映射为 4 类 AC. 每类 AC 具有不同的 AIFS、竞争窗口初始值和竞争窗口最大值. 对于第 $i(i=0,1,2,3)$ 类 $A C$, 其 $A I F S$ 表示为 $A I F S(i)$, 最小竞争窗口为 $C W_{\min }(i)$, 竞争窗口最大值为 $C W_{\max }(i)$. EDCA 机制下数据帧传输示意图如图 1 所示.

从图 1 中可以看出,拥有较小的 AIFS(i)或竞争窗口的 AC 将优先获得无线信道的访问权.在本文中,约定 4 类 AC 按优先级从高到低依次为 AC3,AC2,AC1,AC0.

对应于 4 类 AC, 每个终端建立 4 个 MAC 发送队列. 各种业务的数据帧首先映射到不同的 AC, 放入相应的 
发送队列.每个发送队列采用 DCF(distributed coordination function)机制 ${ }^{[7]}$ 独立竞争信道.在竞争过程中,它们使 用各自的 $\operatorname{AIFS}(i), C W_{\min }(i), C W_{\max }(i)$ 和最大重传次数值.当同一终端内部的多个发送队列之间因竞争信道发生 冲突时, 由一个虚拟调度器(virtual collision handler)选择优先级较高的队列进行发送,而其他低优先级队列的竞 争窗口加倍,重传次数加 1. 当不同终端同时发送数据帧时将发生碰撞,发生碰撞的各个发送队列都要经历重传 过程,与队列优先级高低无关.

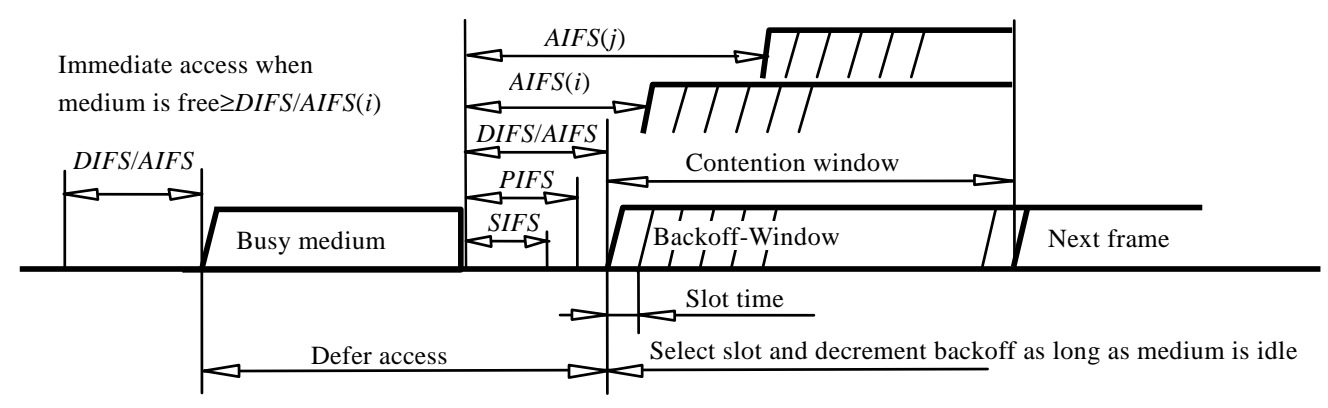

Fig.1 IEEE 802.11e EDCA basic access mechanism

图 1 IEEE 802.11e EDCA 基本访问机制

根据 IEEE 802.11e 的规定,各类 AC 的 AIFS 的取值规则如下:

$$
\operatorname{AIFS}(i)=\operatorname{SIFS}+A(i) \times \sigma, i=0,1,2,3
$$

其中, $A(i)$ 是第 $i$ 类 $\mathrm{AC}$ 的 AIFS 中包含的时隙个数, 具体取值可以参考 IEEE 802.11e 的规定, $\sigma$ 为一个时隙的长度.

EDCA 机制与 DCF 机制一样,同样存在基本模式和 RTS/CTS 模式两种模式.在基本模式下,发送方在竞争 到信道后, 直接发送数据帧, 等待返回 ACK 帧,完成一次数据传输. 在 RTS/CTS 模式下, 发送方在竞争到信道后, 首先利用 RTS 和 CTS 帧请求信道,并预留信道.使用 RTS/CTS 模式可以消除隐终端的影响 ${ }^{[8]}$.

\section{2 信号流图与梅森公式}

信号流图 ${ }^{[9]}$ 是用有向图来描述方程组变量间因果关系的一种图形. 它是一种直观的描述方法, 能够简便地 描述系统, 而且通过梅森公式 ${ }^{[9]}$ 将系统函数与对应的信号流图联系, 可以显著地简化对系统函数的计算, 这对于 复杂的系统函数的计算尤为重要.

梅森公式如下:

$$
H=\frac{1}{\Delta} \sum_{k} g_{k} \Delta_{k}
$$

式中, $H$ 为输入输出系统间的系统函数, $\Delta$ 称为信号流图的特征行列式, 它可以表达为

$$
\Delta=1-\sum_{a} L_{a}+\sum_{b, c} L_{b, c}-\sum_{d, e, f} L_{d, e, f}+\ldots,
$$

其中, $\sum_{a} L_{a}, \sum_{b, c} L_{b, c}, \sum_{d, e, f} L_{d, e, f}$ 分别是所有不同环路的增益之和、所有两两互不接触环路的增益乘积之和、所有 3 个都互不接触环路的增益乘积之和,依此类推 $; k$ 是由源点到终点之间第 $k$ 条前向通路的标号; $g_{k}$ 是由源点到终 点之间第 $k$ 条前向通路的增益; $\Delta_{k}$ 称为第 $k$ 条前向通路特征行列式的余因子,它是除去与第 $k$ 条前向通路相接触 的环路后余下的特征行列式.

在本文提出的模型中,利用信号流图来分析 MAC 接入过程.在信号流图的转移支路上,同时标识转移概率 和所需要的时间. 假设对于某条转移支路,转移概率为 $p$, 完成转移所需时间为 $T$, 则将该支路标识为 $p Z^{T}$. 在此方 式下,利用梅森公式求解得到的系统函数就是 MAC 接入延时的母函数.

\section{2 分析模型}

本文的分析模型遵循以下假设: 
数据帧在尝试发送时发生碰撞的概率是一个常量, 它与已经发生的重传次数无关.

信道为无错信道,不考虑隐终端问题和信道的捕获效应 ${ }^{[8]}$.

对第 $i$ 类 AC, 数据帧最大重传次数为 $m_{i}^{\prime}$, 重传次数超过 $m_{i}^{\prime}$ 的数据帧被丢弃. 竞争窗口最大回退次数为 $m_{i}, m_{i}$ 与 $C W_{\min }(i)$ 和 $C W_{\max }(i)$ 的关系如下:

$$
C W_{\text {max }}(i)=C W_{\text {min }}(i) \times 2^{m_{i}}
$$

网络中终端个数为 $n$,且只考虑饱和情形, 即每个终端中 4 类 AC 均有数据等待发送. 为简化分析, 假设所有 数据帧等长.

只考虑基本模式传输.但是将本文的模型扩展到 RTS/CTS 模式,可得到类似的结论.

\subsection{MAC接入延时}

定义 1. MAC 接入延时是指一个数据帧从到达队列头部开始竞争信道, 直到成功发送完成或者重传次数达 到限制为止被丢弃的时间.

在本文中,我们以随机变量 $T_{s, i}$ 表示第 $i$ 类 AC 的 MAC 接入延时. 根据 IEEE 802.11e 协议的规定,SIFS,DIFS 以及时隙等都以微秒 $(\mu \mathrm{s})$ 为单位, 故而本文中以 $\mu \mathrm{s}$ 作为 MAC 接入延时的时间单位, 此时 $T_{s, i}=k$, 即 $T_{s, i}=k \mu \mathrm{s}$.

记 $p\left(T_{s, i}=k\right)=p_{k}$, 则 $T_{s, i}$ 的母函数表示为

$$
P_{T_{s, i}}(Z)=\sum_{k=0}^{\infty} p_{k} Z^{k}=p_{0} Z^{0}+p_{1} Z^{1}+p_{2} Z^{2}+\ldots
$$

\section{2 回退过程分析}

EDCA 机制中存在 4 类 AC,对于第 $i$ 类 $(i=0,1,2,3) A C$, 可以利用二维 Markov 链模型分析其性能.该模型继承 自文献[2]中的模型, 考虑到数据帧最大重传次数与竞争窗口最大回退次数的不同,对该模型作了一定的扩展:当 重传次数超过竞争窗口最大回退次数时, 竞争窗口将保持为 $C W_{\max }(i)$ 不再增大. 由于各类 AC 具有类似的 MAC 竞争过程,因此可用统一的 Markov 模型建模,通过下标区分不同类的 AC. 对第 $i$ 类 AC, 记 $s_{i}(t)$ 为重传次数, $b_{i}(t)$ 为回退计数器值, 则二维组 $\left(s_{i}(t), b_{i}(t)\right)$ 表示的离散 Markov 状态转移图如图 2 所示.

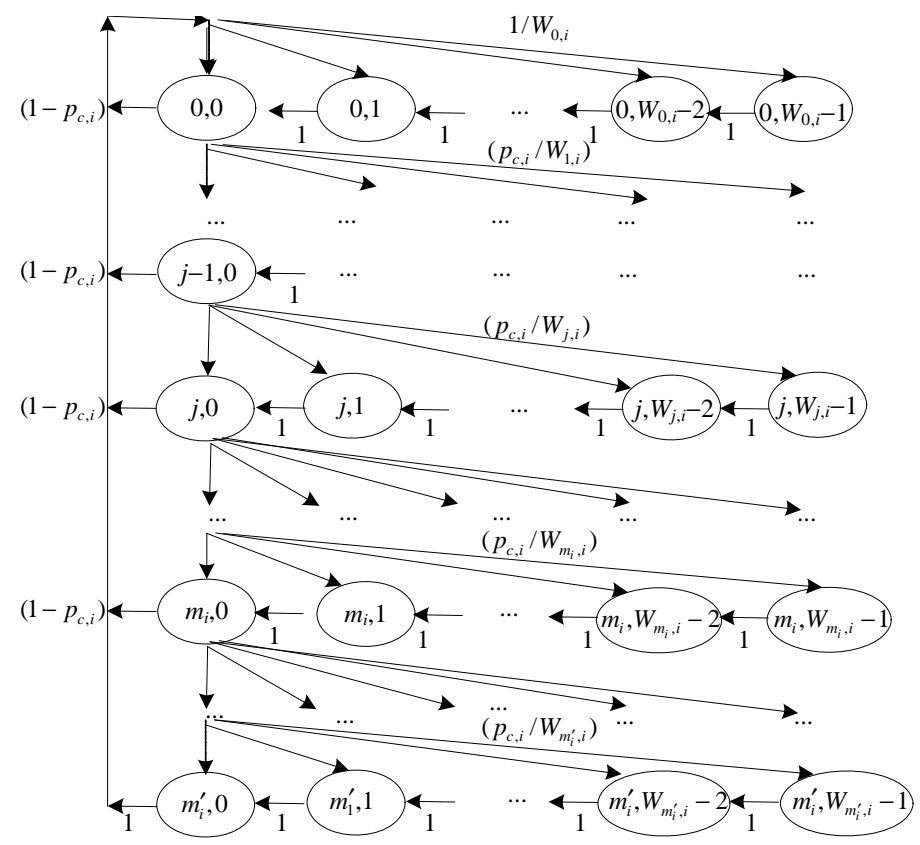

Fig.2 Markov state transition diagram for backoff process of ACi

图 2 第 $i$ 类 AC 回退过程的 Markov 状态转移图 
在图 2 中, 当回退计数器减小到 0 时, 终端将发送数据帧,此时若有其他终端同时发送数据,或者同一终端内 的优先级更高的 AC 发送数据, 就会发生碰撞, 称这一概率为碰撞概率, 记为 $p_{c, i}$, 它代表第 $i$ 类 AC 在发送数据时 发生碰撞的概率. $W_{0, i}=C W_{\min }(i), W_{k, i}\left(0 \leq k \leq m_{i}^{\prime}\right)$ 代表第 $i$ 类 AC 在第 $k$ 次重传时竞争窗口的大小, 其取值如下:

$$
W_{k, i}= \begin{cases}2^{k} W_{0, i}, & 0 \leq k \leq m_{i} \\ 2^{m_{i}} W_{0, i}, & m_{i}<k \leq m_{i}^{\prime}\end{cases}
$$

第 $i$ 类 $\mathrm{AC}$ 的非空一步转移矩阵如下:

$$
\begin{cases}P_{i}\{j, k \mid j, k+1\}=1, & k \in\left[0, W_{j, i}-2\right], j \in\left[0, m^{\prime}\right] \\ P_{i}\{0, k \mid j, 0\}=\left(1-p_{c, i}\right) / W_{0, i}, & k \in\left[0, W_{0, i}-1\right], j \in\left[0, m^{\prime}-1\right] \\ P_{i}\left\{0, k \mid m_{i}^{\prime}, 0\right\}=1 / W_{0, i}, & k \in\left[0, W_{0, i}-1\right] \\ P_{i}\{j, k \mid j-1,0\}=p_{c, i} / W_{j, i}, & k \in\left[0, W_{j, i}-1\right], j \in\left[1, m^{\prime}\right]\end{cases}
$$

令 $b_{j, k, i}=\lim _{t \rightarrow \infty} P_{i}\left\{s_{i}(t)=j, b_{i}(t)=k\right\}\left(j \in\left[0, m_{i}^{\prime}\right], k \in\left[0, W_{j, i}\right], i=0,1,2,3\right)$ 为稳定分布概率. 对任意一个终端中的 第 $i$ 类 AC, 在任意时隙发送数据的概率称为发送概率, 记为 $\tau_{i}$, 则可求得 $\tau_{i}$ 的表达式如下 (具体推导过程见附录):

$$
\tau_{i}=\sum_{j=0}^{m_{i}^{\prime}} b_{j, 0, i}= \begin{cases}\frac{2\left(1-p_{c, i}^{m^{\prime}+1}\right)\left(1-2 p_{c, i}\right)}{1+W_{0, i}-2^{m_{i}} p_{c, i}^{1+m_{i}} W_{0, i}-p_{c, i}\left(2+W_{0, i}\right)-p_{c, i}^{1+m_{i}^{\prime}}\left(1+2^{m_{i}} W_{0, i}\right)+2 p_{c, i}^{2+m_{i}^{\prime}}\left(1+2^{m_{i}} W_{0, i}\right)}, & m_{i}<m_{i}^{\prime} \\ \frac{2\left(1-p_{c, i}^{m^{\prime}+1}\right)\left(1-2 p_{c, i}\right)}{1+W_{0, i}-\left(2 p_{c, i}\right)^{1+m_{i}^{\prime}} W_{0, i}-p_{c, i}\left(2+W_{0, i}\right)-p_{c, i}^{1+m_{i}^{\prime}}+2 p_{c, i}^{2+m_{i}^{\prime}}\left(1+2^{m_{i}^{\prime}} W_{0, i}\right)}, & m_{i}^{\prime} \leq m_{i}\end{cases}
$$

对任一终端而言,该终端在任意时隙发送的概率(即至少有 1 类 AC 发送的概率)为

$$
\tau=1-\prod_{i=0}^{3}\left(1-\tau_{i}\right)
$$

根据碰撞概率的定义, 可得

$$
p_{c, i}=1-(1-\tau)^{n-1} \prod_{j>i}^{3}\left(1-\tau_{j}\right)
$$

对于每一类 AC, $\tau_{i}$ 与 $p_{c, i}$ 都满足式(7)和式(9). 由式(9)可知,各类 AC 之间的发送概率和碰撞概率相互关联, 因此需要将它们联立起来, 经过迭代求解, 求得 $\tau_{i}$ 与 $p_{c, i}$ 的值. 这两个值为后续的接入延时分析提供了依据.

\section{3 延时分析}

在回退过程中, 每个 AC 都监听信道, 如果信道空闲, 则每隔一个空闲时隙, 回退计数器减 1 ; 如果信道上有其 他 $\mathrm{AC}$ 发送数据, 则计数器将挂起, 设计数器挂起的概率为挂起概率, 记为 $p_{f, i}$, 它意味着其他终端或者本终端的其 他 AC 有数据传输. 注意, 它与碰撞概率 $p_{c, i}$ 的含义不同.

$$
p_{f, i}=1-(1-\tau)^{n-1} \prod_{j=0, j \neq i}^{3}\left(1-\tau_{j}\right)
$$

在这些传输中,成功的概率为 $p_{s u c, i}$, 发生冲突的概率为 $p_{f, i}-p_{s u c, i}, p_{s u c, i}$ 可表示为

$$
p_{\text {suc }, i}=\sum_{j=0, j \neq i}^{3}\left\{n \tau_{j}\left(1-\tau_{j}\right)^{n-1}\left(1-\tau_{i}\right)^{n-1}\left(\prod_{k=0, k \neq i, j}^{3}\left(1-\tau_{k}\right)^{n}\right)\right\}+(n-1) \tau_{i}\left(1-\tau_{i}\right)^{n-2} \prod_{j=0, j \neq i}^{3}\left(1-\tau_{j}\right)^{n}
$$

设成功发送所需时间为 $T_{s u c, i}$, 发生冲突耗费时间为 $T_{c o l, i}, T_{s u c, i}$ 的母函数为 $S_{t, i}(Z), T_{c o l, i}$ 的母函数为 $C_{t, i}(Z)$. 根据 IEEE 802.11e 协议, $T_{s u c, I}, T_{c o l, I}, S_{t, i}(Z), C_{t, i}(Z)$ 可分别计算如下:

$$
\left\{\begin{array}{l}
T_{\text {suc }, i}=H+T_{\text {Load }}+T_{A C K}+\text { SIFS }+ \text { AIFS }(i) \\
T_{\text {col }, i}=H+T_{\text {Load }}+\text { EIFS } \\
S_{t, i}(Z)=Z^{T_{\text {suc }, i}} \\
C_{t, i}(Z)=Z^{T_{\text {col }, i}}
\end{array}\right.
$$

其中, $H=P H Y_{h d r}+M A C_{h d r}, T_{\text {load }}$ 为传输数据帧净荷所需的时间, $T_{A C K}$ 为发送 ACK 所需的时间. 
在计数器的一步回退过程中, 以概率 $p_{f, i}$ 挂起计数器, 以概率 $1-p_{f, i}$ 递减计数器. 挂起计数器分为两种情形: 一 种情形为某个终端中的 AC $i$ 成功发送数据, 它发生的概率为 $p_{s u c, i}$, 所需时间为 $T_{s u c, i}$, 则此时除此特定终端的 AC $i$ 之外的任一 $\mathrm{AC}$ 的回退计数器都非零, 否则, 由 CSMA/CA 机制, 必然会发生冲突而导致无法成功发送数据. 因此, 发送完成后所有 $\mathrm{AC}$ 的回退计数器都非零, 下一步将以概率 1 递减回退计数器, 所需时间为 $\sigma$, 另一种情形为两个 或两个以上 AC 同时发送导致冲突, 冲突的概率为 $p_{f, i}-p_{s u c, i}$, 冲突时间为 $T_{c o l, i}$, 此时, 所有其他 AC 的回退计数器都 非零. 因此, 在冲突过后, 所有 AC 的回退计数器都非零, 下一步也将以概率 1 递减回退计数器, 所需时间为 $\sigma$. 由此 可得一步回退的信号流图,如图 3 所示.

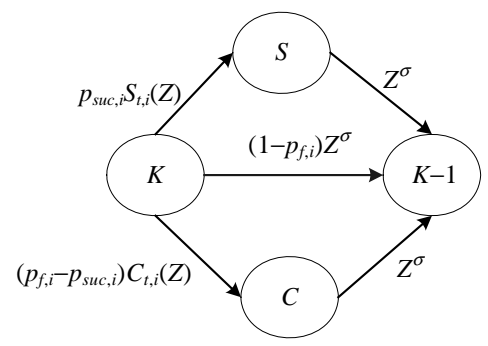

Fig.3 Signal flow graph for one step backoff

图 3 一步回退信号流图

从开始回退到最终成功发送或者重传次数超限被丢弃的总信号流图如图 4 所示.

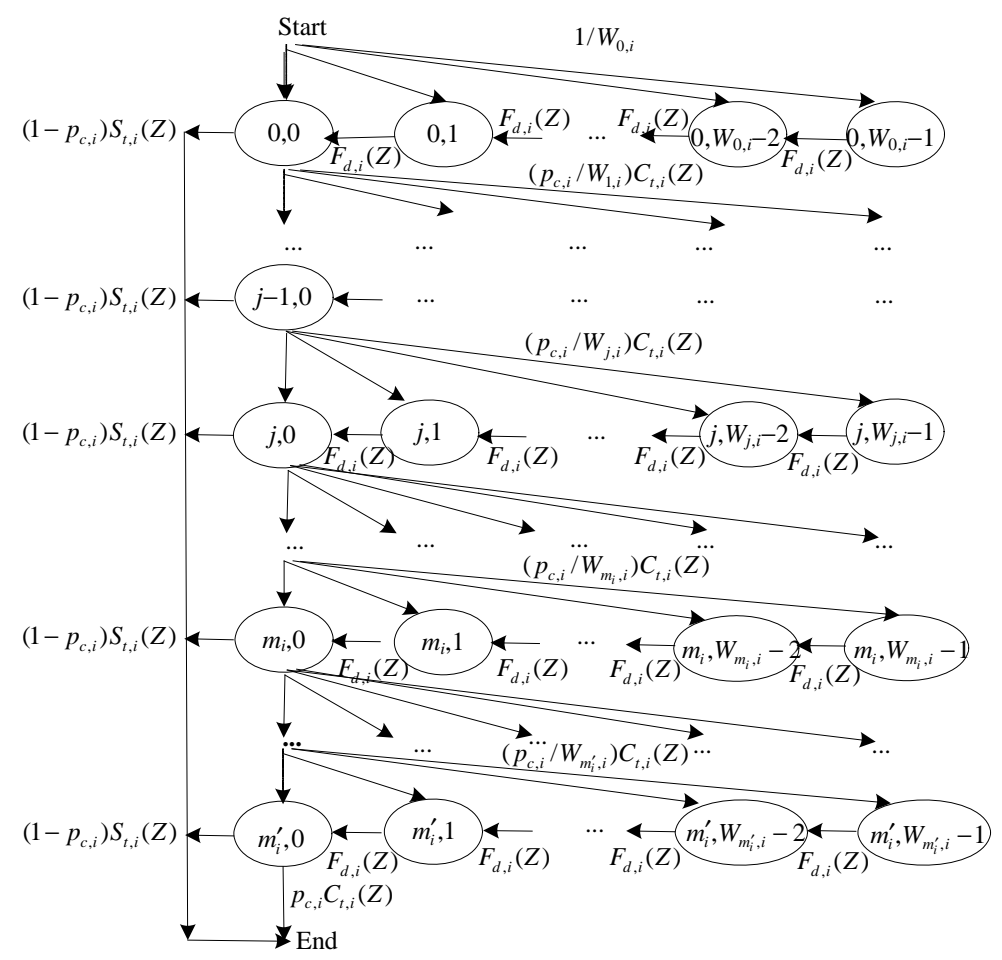

Fig.4 Signal flow graph for MAC access process of ACi

图 4 第 $i$ 类 AC 的 MAC 接入过程信号流图

利用梅森公式, 从图 3 可得一步回退过程延时的母函数为

$$
F_{d, i}(Z)=\left(1-p_{f, i}\right) Z^{\sigma}+p_{s u c, i} S_{t, i}(Z) Z^{\sigma}+\left(p_{f, i}-p_{s u c, i}\right) C_{t, i}(Z) Z^{\sigma}
$$


在图 4 中, 对于各个回退阶段, 它们的延时的母函数为

$$
G S_{k, i}(Z)=\left(\sum_{j=0}^{W_{k, i}-1} F_{d, i}(Z)^{j}\right) / W_{k, i}, 0 \leq k \leq m_{i}^{\prime}
$$

记 $G_{k, i}(Z)=\prod_{j=0}^{k} G S_{j, i}(Z)$, 表示第 $i$ 类 AC 前 $k$ 个回退阶段的总延时的母函数.

接入延时的母函数可表示为

$$
P_{T_{s, i}}(Z)=\left(1-p_{c, i}\right) S_{t, i}(Z) \sum_{j=0}^{m_{i}^{\prime}}\left(\left(p_{c, i} C_{t, i}(Z)\right)^{j} G_{j, i}(Z)\right)+\left(p_{c, i} C_{t, i}(Z)\right)^{m_{i}^{\prime}+1} G_{m_{i}^{\prime}, i}(Z)
$$

将该式展开,即可得第 $i$ 类 AC 的 MAC 接入延时 $T_{s, i}$ 的概率分布:

$$
P_{T_{s, i}}(Z)=\sum_{k=0}^{\infty} p\left(T_{s, i}=k\right) Z^{k}
$$

由式(16)可得 MAC 接入延时的概率分布, 并且由母函数的性质可得其均值和方差分别为

$$
\left\{\begin{array}{l}
E\left[T_{s, i}\right]=P_{T_{s, i}}^{\prime}(1) \\
\operatorname{VAR}\left[T_{s, i}\right]=P_{T_{s, i}}^{\prime \prime}(1)+P_{T_{s, i}}^{\prime}(1)-\left[P_{T_{s, i}}^{\prime}(1)\right]^{2}
\end{array}\right.
$$

\section{3 模型验证}

采用 NS-2 $2^{[10]}$ 进行了多次仿真实验,得到各类 AC 在不同终端数目情形下 MAC 接入延时的分布,并与分析 模型的结果进行比较,验证本文的分析模型.仿真实验中的参数设置见表 1 .

当 $n=5$ 时,根据分布模型计算得出的各类 AC 的 MAC 接入延时的完全概率分布如图 5(a) 图 5(d)所示.

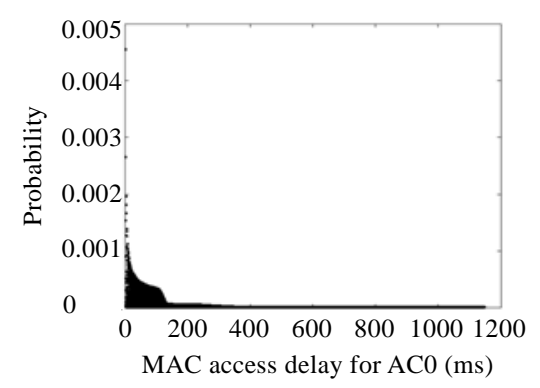

(a) AC0

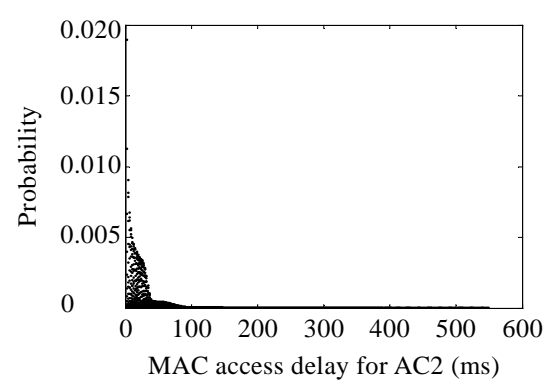

(c) AC2

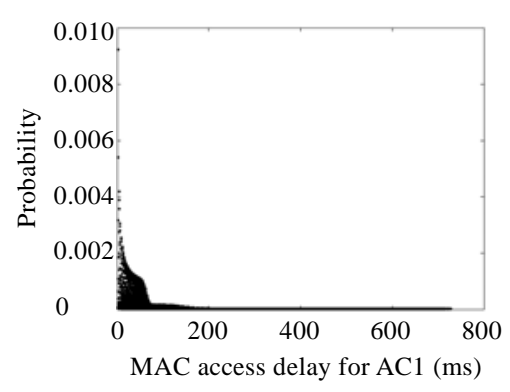

(b) AC1

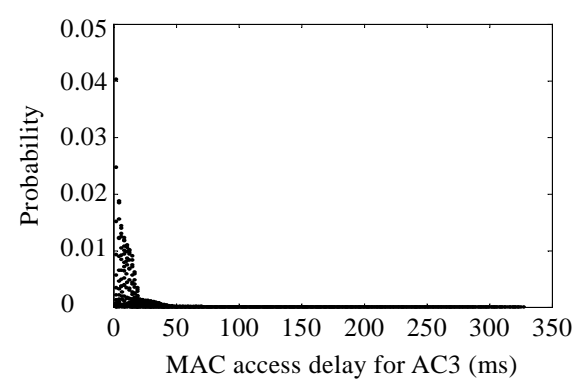

(d) AC3

Fig.5 Full probability distribution of MAC access delay of all the ACs

图 5 各类 AC 的 MAC 接入延时完全概率分布

获取 MAC 接入延时在各个时间段的概率分布, 可对 MAC 接入过程有更为清晰和直接的认识. 为此, 将上述 离散时间点的概率数据按照时间段汇总, 得到 MAC 接入延时在不同时间段的分布. 在仿真实验中,统计 24 小时 
内各类 AC 的 MAC 接入延时的分布并汇总数据, 可以得到 MAC 接入延时分布的仿真结果. 将分析模型的结果 和仿真结果以直方图的形式绘制在一起,其概率分布图如图 6(a) 图 6(d)所示.

从图 6 可以看出,本文所提出的分析模型获得的 MAC 接入延时与仿真得到的结果吻合良好,这证明了该模 型的正确性.对于不同的终端数目,多次运行仿真,均得到类似的结论.

Table 1 Parameters for IEEE 802.11e

表 1 IEEE 802.11e 的参数

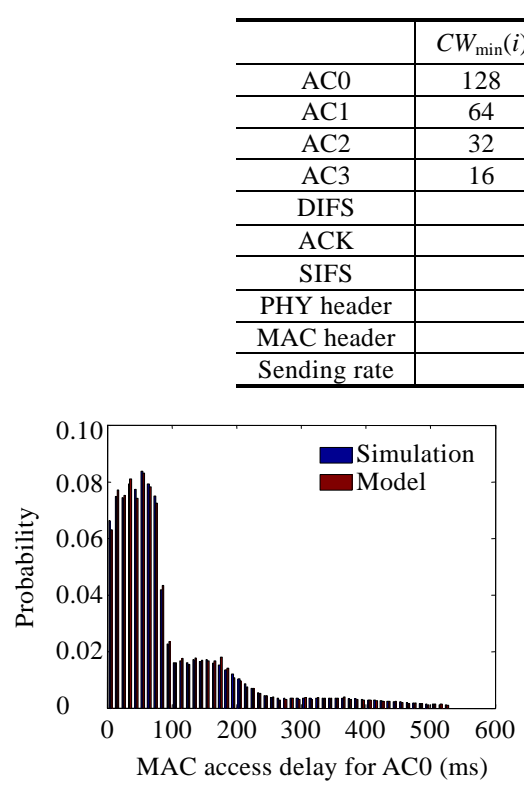

(a) AC0

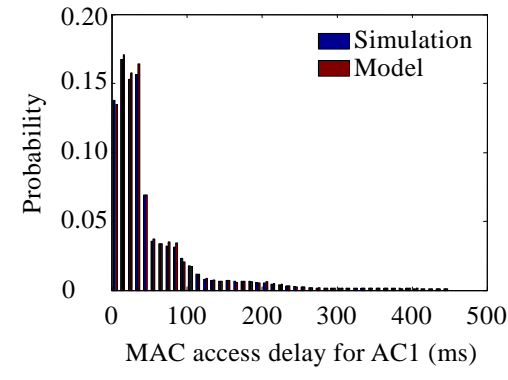

(b) AC1

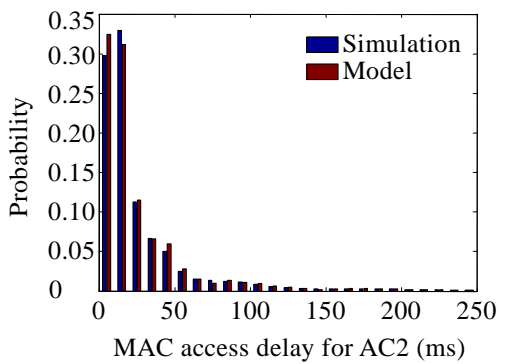

(c) AC2

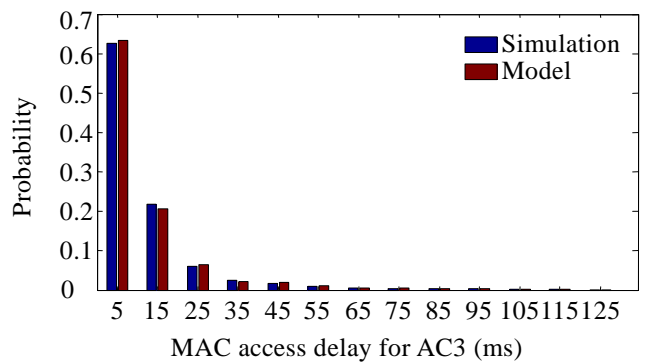

(d) AC3

Fig.6 Probability distribution of MAC access delay of all the ACs (gathered by time interval) 图 6 各类 AC 的 MAC 接入延时概率分布(按时间段汇总)

\section{4 分析结果}

\section{1 终端数目对性能的影响}

根据分析模型, 在表 1 所示的系统参数设置下, 发送概率 $\tau$ 、冲突概率 $P_{c}$ 、挂起概率 $P_{f}$ 和接入延时 Delay 随 终端数 $n$ 的变化曲线如图 7 所示. 由图可知, 对于相同的 $n$ 值, AC 的优先级越高, 则其发送概率越大, 碰撞概率和 挂起概率就越小, 相应的 MAC 接入延时也就越小.对于每类 AC, 随着 $n$ 值的增大, 发送概率将减小, 碰撞概率、 挂起概率和 MAC 接入延时将增大. 


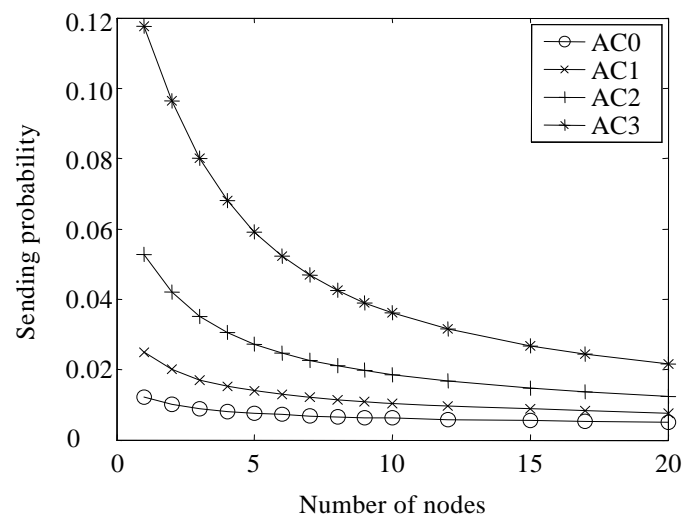

(a) Sending probability

(a) 发送概率

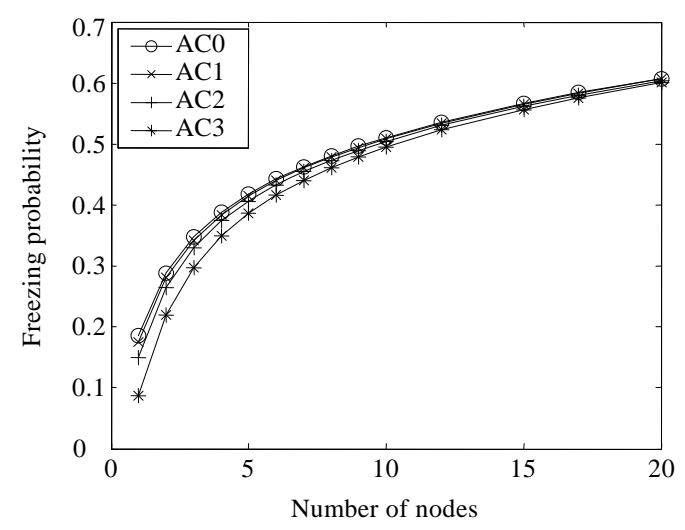

(c) Freezing probability

(c) 挂起概率

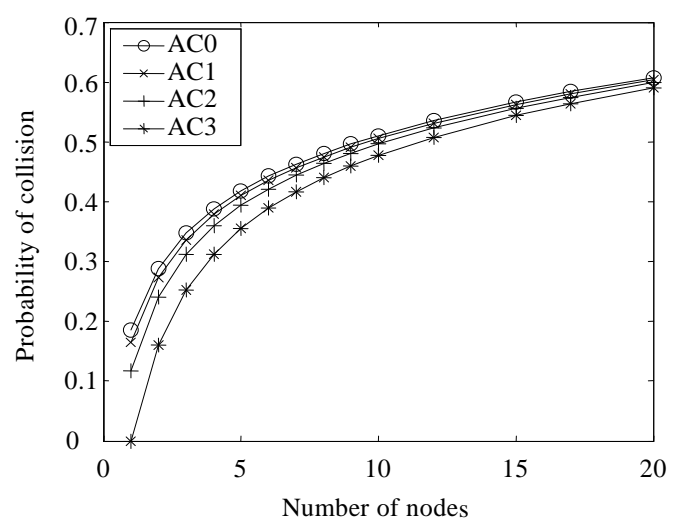

(b) Collision probability

(b) 冲突概率

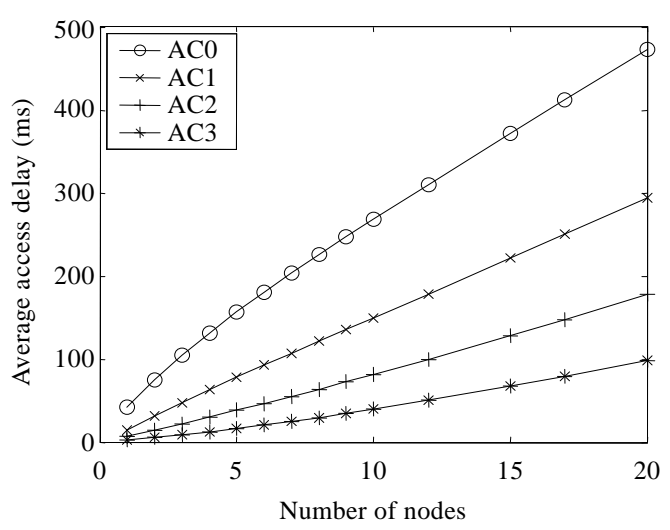

(d) MAC access delay

(d) MAC 接入延时

Fig.7 Relationship among number of stations and sending probability, collision probability, freezing probability and MAC access delay

图 7 发送概率、冲突概率、挂起概率和 MAC 接入延时与终端数的关系

\subsection{MAC接入延时成因分析}

利用本文的分析模型,可以揭示 MAC 接入延时的成因.

以包含 5 个终端的 WLAN 为例, 参数设置见表 1 , 计算各 AC 在不同回退阶段的 MAC 接入延时均值,结果 列于表 2 .

Table 2 Mean value of delay for all backoff stages of four ACs

(ms)

表 24 类 AC 各回退阶段延时均值

(ms)

\begin{tabular}{lcccccccc}
\hline & Stage 0 & Stage 1 & Stage 2 & Stage 3 & Stage 4 & Stage 5 & Stage 6 & Stage 7 \\
\hline AC3 $\left(C W_{\min }=16\right)$ & 4.3 & 8.7 & 17.9 & 36 & 72 & 145 & 290 & 290 \\
AC2 $\left(C W_{\min }=32\right)$ & 9.4 & 19.2 & 38.7 & 78 & 155 & 311 & 311 & 311 \\
AC1 $\left(C W_{\min }=64\right)$ & 19.7 & 39.8 & 79.9 & 160 & 321 & 321 & 321 & 321 \\
AC0 $\left(C W_{\min }=128\right)$ & 40.6 & 81.4 & 163.2 & 327 & 327 & 327 & 327 & 327 \\
\hline
\end{tabular}

由表 2 可知, $C W_{\min }$ 值决定了各 $\mathrm{AC}$ 在不同回退阶段的延时. 因为数据帧在重传时,其总延时为所经过的各回 退阶段延时之和, 因此, $C W_{\text {min }}$ 值进一步决定了各 $\mathrm{AC}$ 在不同重传次数下的延时. 以 $\mathrm{AC}$ 为例, 在不同重传次数下, 按时间段汇总的接入延时分布概率曲线如图 8 所示. 由图 8 可知,随着重传次数的增大,延时分布的范围将逐渐 
增大, 延时均值也随之增大.可计算得出,0 次重传时 (一次传输即成功), 延时主要分布在 $0 \mathrm{~ms} 40 \mathrm{~ms}$ 之间,均值为 $4.3 \mathrm{~ms}$; 当重传次数达到 7 次时,延时主要分布在 $270 \mathrm{~ms} 1470 \mathrm{~ms}$ 之间,均值为 $865 \mathrm{~ms}$.

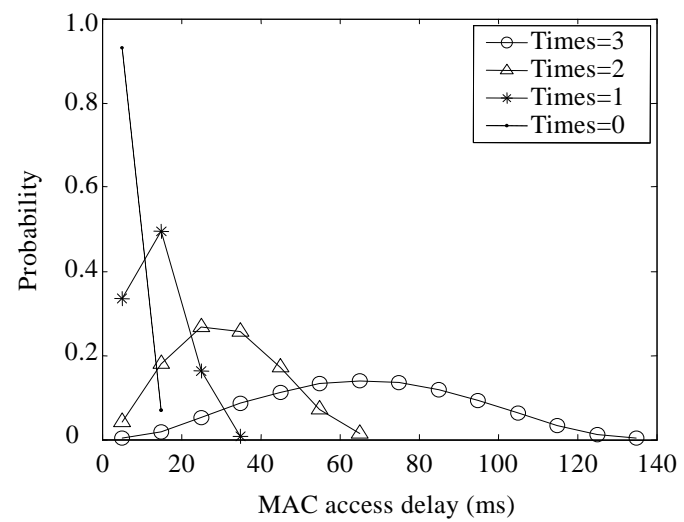

(a) Retransmission times $=0,1,2,3$

(a) 重传次数 $=0,1,2,3$

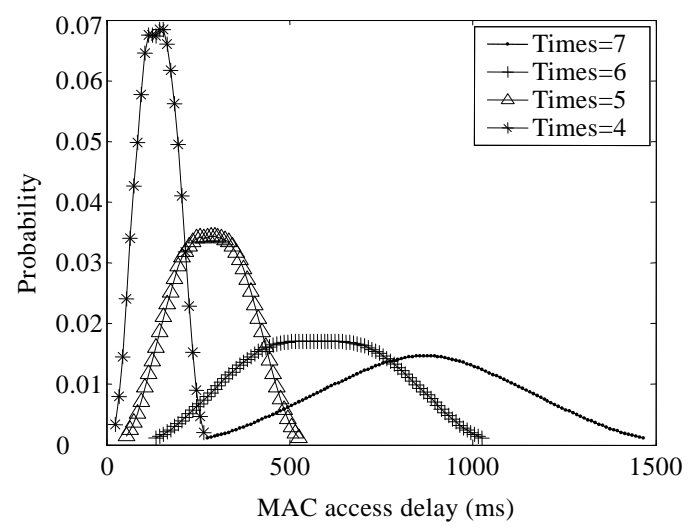

(b) Retransmission times $=4,5,6,7$

(b) 重传次数 $=4,5,6,7$

Fig.8 MAC access delay distribution with different retransmission times

图 8 不同重传次数下的 MAC 接入延时分布

在回退过程中,碰撞概率决定重传特性. 根据式(15), 总的 MAC 接入延时为各重传次数下延时的加权平均, 权值为取该重传次数的概率. 以网络中 AC3 为例来说明碰撞概率对延时的影响. 当 $n=5$ 时, AC3 的碰撞概率为 0.356, 数据帧经过 0 7 次重传成功传输的概率分别为 $0.644,0.229 \quad 26,0.08162,0.029 \quad 06,0.01034,0.00368$, $0.00131,0.00073$, 一次传输成功的均值为 $4.3 \mathrm{~ms}$, 而总的 MAC 接入延时均值为 $16.2 \mathrm{~ms}$, 其中, 由于 1 次或多次重 传而耗费的时间占总延时的 $82.9 \%(1-(4.3 \times 0.644 / 16.2)=82.9 \%)$. 当 $n=20$ 时,AC3 的碰撞概率为 0.591 , 经过 $0 \sim 7$ 次重传成功传输的概率分别为 0.409 0,0.241 72,0.142 86,0.084 43,0.049 90,0.029 49,0.017 43,0.025 18, 一次传输 成功的均值为 $6.5 \mathrm{~ms}$, 而总的 MAC 接入延时均值将达到 $98.6 \mathrm{~ms}$, 其中, 由于 1 次或多次重传而耗费的时间占总延 时的 97.3\%. 由此可见, 随着碰撞概率的增大, 数据帧将经历更多的重传, 进而使得总 MAC 接入延时增大. 而由上 一节的分析可知,当网络中终端数增多时, 碰撞概率随之增大, 从而导致总 MAC 接入延时增大.

因此,一方面, $C W_{\min }$ 值决定了各重传次数下的延时值, 从而影响总 MAC 接入延时; 另一方面, 随着终端数的 增多,碰撞概率随之增大,数据帧经历更多的重传,这些重传导致更大的 MAC 接入延时.

\subsection{AIFS 值对性能的影响}

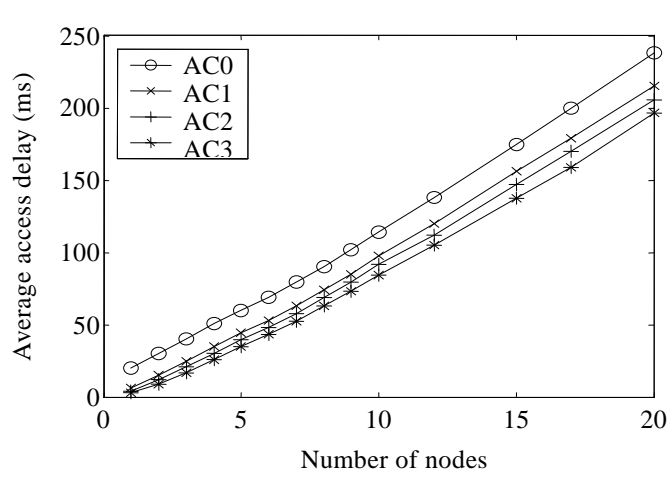

Fig.9 MAC access delay vs. AIFS

图 9 MAC 接入延时 vs. AIFS
在 EDCA 机制中,通过为不同的 AC 类别设置 AIFS 值,可以赋予各类 AC 不同的优先级访问信道,较小 AIFS 值的 AC 可以优先获得信道访问权.为反映 AIFS 值对不 同 AC 类别延时的影响,仿真参数设置如下:将各类 AC 的 $C W_{\min }$ 值设置为相同的值 16 以消除初始竞争窗口不同的 影响,其余的参数设置与表 1 相同, 即 AC0,AC1,AC2, AC3 的 AIFS 取值依次为 $30 \mu \mathrm{s}, 70 \mu \mathrm{s}, 90 \mu \mathrm{s}, 110 \mu \mathrm{s}$. 再次运行仿 真,得到不同类 AC 的 MAC 接入延时曲线,如图 9 所示. 由图可知, 在该参数设置下, 不同的 AIFS 值对 MAC 接入 延时有一定的影响, 优先级最高的 AC3 的延时最小, 优先 级最低的 AC0 的延时最大, 但是各类 AC 之间 MAC 接入 延时值差异比较小. 这是因为根据 EDCA 的取值规 
则,AIFS 的取值范围一般在 $30 \mu \mathrm{s} \sim 110 \mu \mathrm{s}$ 之间, 相对回退时间(数百 $\mu \mathrm{s}$ 到数十 $\mathrm{ms}$ ) 而言, 这是一个比较小的值, 虽然 在多次重传或信道挂起后等待重新获得信道的过程中需要经历多次 AIFS, 但所造成的总的 MAC 接入延时的变 化还是比较小的.当 AIFS 的取值增大时,各类 AC 之间的 MAC 接入延时相应地将有较大的变化.

\subsection{MAC接入延时方差分析}

根据分析模型得到的概率分布,利用式(17), 可以进一步计算得到 MAC 接入延时的方差和标准差.

变异系数(deviation coefficient)为标准差与均值之商. 它是排队理论中的重要指标. 对于负指数分布, 其变异 系数为 1 .对于 $n=5$ 和 $n=20$ 两种情形,计算得到均值、标准差和变异系数的结果见表 3 .

Table 3 Mean/standard deviation/deviation coefficient for four ACs

表 34 类 AC 的均值、标准差和变异系数

\begin{tabular}{c|c|c|c|c}
\hline \multicolumn{2}{c|}{} & Mean (ms) & Standard deviation (ms) & Deviation coefficient \\
\hline \multirow{5}{*}{$n=5$} & AC0 & 156 & 244 & 1.56 \\
& AC1 & 78 & 161 & 2.06 \\
& AC2 & 38 & 93 & 2.45 \\
& AC3 & 16 & 41 & 2.56 \\
\hline \multirow{5}{*}{$n=20$} & AC0 & 473 & 689 & 1.45 \\
& AC1 & 295 & 535 & 1.81 \\
& AC2 & 178 & 389 & 2.18 \\
& AC3 & 99 & 255 & 2.57 \\
\hline
\end{tabular}

在网络中终端数从 2 变化到 20 的过程中, 4 类 AC 变异系数的变化趋势如图 10 所示.从该图可知,在终端数 目较少的情形下, 变异系数首先快速增大, 当终端数目增大到一定数值时, 变异系数变化趋于平缓.MAC 接入延 时的标准差通常大于均值,变异系数大部分集中在 1.5 3 之间.因此,根据排队分析理论,在延时分析中使用负指 数分布作为 MAC 接入延时的分布来进行计算, 将导致计入的方差偏小, 从而得到的平均等待队长和平均排队 时间都偏小,这会导致估算出的系统服务能力大于系统的实际服务能力. 在排队分析中应该注意并予以修正.

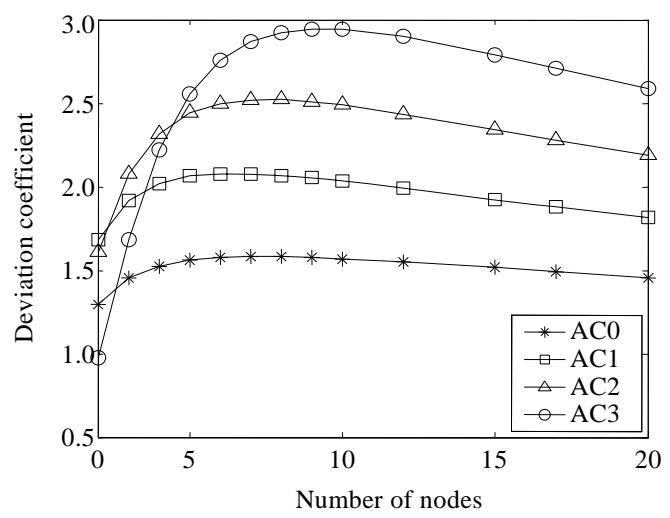

Fig.10 Deviation coefficient

图 10 变异系数

\section{5 结论和未来的工作}

本文提出的分析模型准确地分析了无线局域网中 EDCA 机制的 MAC 接入延时,得到了延时的概率分布以 及相关的数字特征. 经过仿真实验, 验证了本文模型的正确性. 分析表明, 随着终端数目的增多, 发送概率将减小, 碰撞概率和挂起概率将增大,MAC 接入延时也将增大. 在影响 MAC 接入延时的参数中, 初始竞争窗口的大小对 不同类 AC 的 MAC 接入延时的影响较大,而对 AIFS 的值影响有限.最后, 通过标准差和变异系数分析, 指出采用 负指数分布作为 MAC 接入延时的分布会导致较大的误差.

这种准确的性能分析为后续研究改进系统性能的途径提供了基础.下一步工作将结合排队分析,研究适合 
于 WLAN 特性的缓冲管理和调度机制.

\section{References:}

[1] IEEE WG. Draft Supplement to Standard for Telecommunications and Information Exchange Between Systems-LAN/MAN Specific Requirements-Part 11: Wireless Medium Access Control (MAC) and Physical Layer Specifications: Medium Access Control (MAC) Enhancements for Quality of Service (QoS). IEEE 802.11e/Draft D5.0, 2003.

[2] Bianchi G. Performance analysis of the IEEE 802.11 distributed coordination function. IEEE JSAC, 2000,18(3):535-547.

[3] Wu HT, Cheng SD, Peng Y, Long KP, Ma J. IEEE 802.11 distributed coordination function (DCF): Analysis and enhancement. In: Proc. of the IEEE Int'l Conf. on Communications (ICC 2002). New York: IEEE Press, 2002. 605-609.

[4] Xiao Y. An analysis for differentiated services in IEEE 802.11 and IEEE 802.11e wireless LANs. In: Proc. of the 24th Int'l Conf. on Distributed Computing Systems (ICDCS 2004). Tokyo: IEEE Press, 2004. 32-39.

[5] Sheng LZ, Huangfu W, Lei W, Wu ZM. Performance study of real-time traffic in wireless local area network with different kind of stations. Journal on Communications, 2006,27(4):16-22 (in Chinese with English abstract).

[6] Zhai HQ, Kwon YG, Fang YG. Performance analysis of IEEE 802.11 MAC protocols in wireless LANs. Wireless Communications and Mobile Computing, 2004,4(8):917-931.

[7] IEEE Standard for Wireless LAN Medium Access Control (MAC) and Physical (PHY) Layer Specifications. IEEE std 802.11-1999, 1999. http://ieeexplore.ieee.org/iel5/9543/30234/01389197.pdf?tp=\&isnumber=30234\&arnumber=1389197

[8] Kim JH, Lee JK. Capture effects of wireless CSMA/CA protocols in Rayleigh and shadow fading channels. IEEE Trans. on Vehicular Technology, 1999,48(4):1277-1286.

[9] Zhang HQ, Xu XY, Zhao ZJ. Signal and System Analysis. Beijing: China Machine Press, 2006. 284-288 (in Chinese).

[10] http://www.isi.edu/nsnam/ns/

\section{附中文参考文献:}

[5] 盛凌志,皇甫伟,雷文,吴志美.多类别终端无线局域网实时业务性能分析.通信学报,2006,27(4):16-22.

[9] 张华清,许信玉,赵志军.信号与系统分析.北京:机械工业出版社,2006.284-288.

\section{附录. $\tau_{i}$ 的推导过程.}

由 Markov 状态转移图可得

$$
\begin{aligned}
& b_{j-1,0, i} \cdot p_{c, i}=b_{j, 0, i} \text {, } \\
& b_{j, 0, i}=p_{c, i}^{j} b_{0,0, i} . \\
& b_{j, k, i}=\frac{W_{j, i}-k}{W_{j, i}} \cdot\left\{\begin{array}{ll}
\left(1-p_{c, i}\right) \sum_{k=0}^{m_{i}^{\prime}-1} b_{k, 0, i}+b_{m_{i}^{\prime}, 0, i}, & j=0 \\
p_{c, i} b_{j-1,0, i}, & 0<j<m_{i}^{\prime}
\end{array},\right. \\
& b_{j, k, i}=\frac{W_{j, i}-k}{W_{j, i}} b_{j, 0, i} . \\
& 1=\sum_{j=1}^{m_{i}^{\prime}} \sum_{k=0}^{W_{j, i}-1} b_{j, k, i}=\sum_{j=0}^{m_{i}^{\prime}} b_{j, 0, i} \sum_{k=0}^{W_{j, i}-1} \frac{W_{j, i}-k}{W_{j, i}}=\sum_{j=0}^{m_{i}^{\prime}} b_{j, 0, i} \frac{W_{j, i}+1}{2}=\frac{b_{0,0, i}}{2} \sum_{j=0}^{m_{i}^{\prime}} p_{c, i}^{j} \frac{W_{j, i}+1}{2} \\
& =\left\{\begin{array}{ll}
\frac{b_{0,0, i}}{2}\left(\sum_{j=0}^{m_{i}-1} p_{c, i}^{j}\left(2^{j} W_{0, i}+1\right)+\sum_{j=m_{i}}^{m_{i}^{\prime}} p_{c, i}^{j}\left(2^{m_{i}} W_{0, i}+1\right)\right), & m_{i}<m_{i}^{\prime} \\
\frac{b_{0,0, i}}{2}\left(\sum_{j=0}^{m_{i}^{\prime}} p_{c, i}^{j}\left(2^{j} W_{0, i}+1\right)\right), & m_{i}^{\prime} \leq m_{i}
\end{array},\right.
\end{aligned}
$$

化简可得 


$$
b_{0,0, i}=\left\{\begin{array}{ll}
\frac{2\left(1-p_{c, i}\right)\left(1-2 p_{c, i}\right)}{1+W_{0, i}-2^{m_{i}} p_{c, i}^{1+m_{i}} W_{0, i}-p_{c, i}\left(2+W_{0, i}\right)-p_{c, i}^{1+m_{i}^{\prime}}\left(1+2^{m_{i}} W_{0, i}\right)+2 p_{c, i}^{2+m_{i}^{\prime}}\left(1+2^{m_{i}} W_{0, i}\right)}, & m_{i}<m_{i}^{\prime} \\
\frac{2\left(1-p_{c, i}\right)\left(1-2 p_{c, i}\right)}{1+W_{0, i}-\left(2 p_{c, i}\right)^{1+m_{i}^{\prime}} W_{0, i}-p_{c, i}\left(2+W_{0, i}\right)-p_{c, i}^{1+m_{i}^{\prime}}+2 p_{c, i}^{2+m_{i}^{\prime}}\left(1+2^{m_{i}^{\prime}} W_{0, i}\right)}, & m_{i}^{\prime} \leq m_{i}
\end{array} .\right.
$$

从而 $\tau_{i}=\sum_{k=0}^{m_{i}^{\prime}} b_{k, 0, i}=\left\{\begin{array}{ll}\frac{2\left(1-p_{c, i}^{m_{i}^{\prime}+1}\right)\left(1-2 p_{c, i}\right)}{1+W_{0, i}-2^{m_{i}} p_{c, i}^{1+m_{i}} W_{0, i}-p_{c, i}\left(2+W_{0, i}\right)-p_{c, i}^{1+m_{i}^{\prime}}\left(1+2^{m_{i}} W_{0, i}\right)+2 p_{c, i}^{2+m_{i}^{\prime}}\left(1+2^{m_{i}} W_{0, i}\right)}, m_{i}<m_{i}^{\prime} \\ \frac{2\left(1-p_{c, i}^{m_{i}^{\prime}+1}\right)\left(1-2 p_{c, i}\right)}{1+W_{0, i}-\left(2 p_{c, i}\right)^{1+m_{i}^{\prime}} W_{0, i}-p_{c, i}\left(2+W_{0, i}\right)-p_{c, i}^{1+m_{i}^{\prime}}+2 p_{c, i}^{2+m_{i}^{\prime}}\left(1+2^{m_{i}^{\prime}} W_{0, i}\right)}, & m_{i}^{\prime} \leq m_{i}\end{array}\right.$.

这就是式(7).

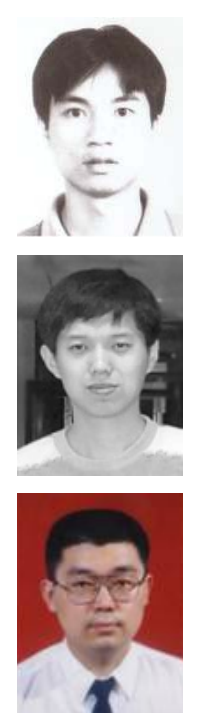

周新运 $(1979-)$, 男, 湖南邵阳人, 博士, 助 理研究员, 主要研究领域为无线通信技术, 无线网络 QoS.

皇甫伟(1975-), 男, 博士, 助理研究员, 主 要研究领域为光网络, 无线网络, 传感器 网络.

孙利民(1968-), 男, 博士, 研究员,CCF 高 级会员, 主要研究领域为无线网络, 传感器 网络.

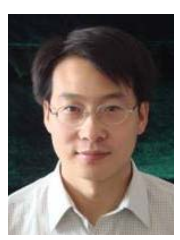

王显雷(1974-), 男,博士, 主要研究领域为 多媒体网络通信,网络服务质量.

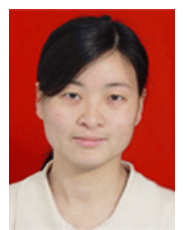

李凯慧(1980-), 女, 博士, 主要研究领域为 视频传输,多媒体通信. 